\title{
ARTICLE
}

\section{Competitive light emission from excited electron and proton systems in polyaniline doped with p-toluenesulfonic acid when electrically powered}

Received 00th January 20xx,

\author{
Jerzy J. Langer*a ${ }^{*}$ Katarzyna C. Ratajczak***, Ewelina Frąckowiak ${ }^{* *}$ and Sebastian Golczak
}

Accepted 00th January 20xx

DOI: $10.1039 / \times 0 \times x 00000 x$

Polyaniline doped with p-toluenesulfonic acid (PANI / PTSA), in which electrons and protons are excited when electrically powered, emits light in two different ranges in a competitive manner. Efficient energy transfer from electrons to protons and excitation of the proton system, resulting in less dissipation of the supplied energy, leads to the emission of higherenergy photons, and more efficiently than an excited polyaniline electron system (for wet PANI / PTSA), when electrically powered. The water proton system successfully competes with the electron system in the wet PANI / PTSA, capturing the supplied electrical energy, which results in intense blue shift light emission.

\section{Introduction}

The emission of light by electrically powered conductive polymers has focused our interests for over ten years. We described polyaniline LEDs with non-linear effects, including stimulated Raman scattering ${ }^{1}$ and polyaniline lasing ${ }^{2}$, but also electro luminescence of polypirrole ${ }^{3}$.

On the other hand, we have discovered emission in the entire range of UV-VIS-NIR due to the excitation of protons in the protonic analog of the $p-n$ junction (protonic LED $^{4}$ ), formed in water as a protonic semiconductor, appropriately doped ${ }^{5,6}$. Now, we describe the system (polyaniline doped with $p$ toluenesulfonic acid, PANI/PTSA), where electrons and protons are excited when electrically powered, emitting the light in different range, on a competitive way.

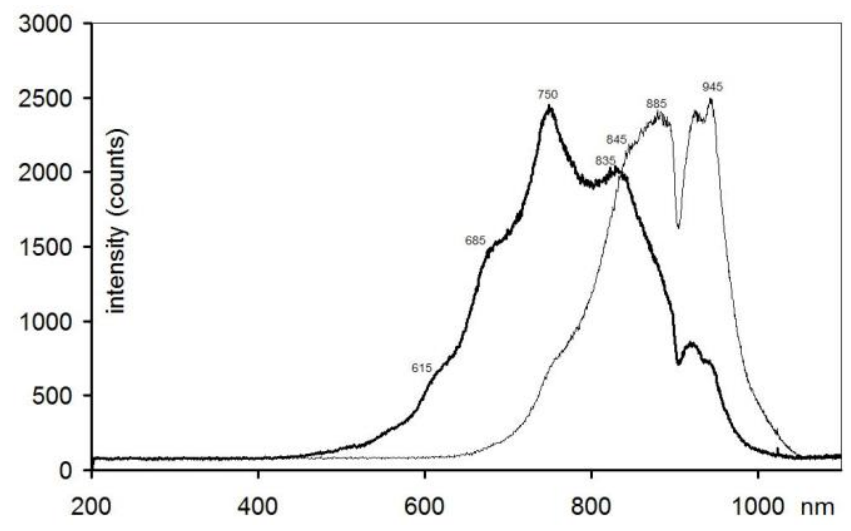

Fig. 1a. Comparison of PANI / PTSA electroluminescence spectra in the dry state (right spectrum) and in the presence of water (left spectrum).

\footnotetext{
a. Mickiewicz University in Poznań, Faculty of Chemistry, Laboratory for Materials Physicochemistry and Nanotechnology, Uniwersytetu

Poznańskiego 6, 61-614 Poznań, Poland

${ }^{*}$ Corresponding author: orsel@sigmaxi.net

** Presently: Ewelina Ludera

*** Presently: SZKOŁA GŁÓWNA GOSPODARSTWA WIEJSKIEGO W WARSZAWIE

Institute of Biology, Department of Physics and Biophysicsul. Nowoursynowska 159, 02-776 Warsaw, Poland
} 


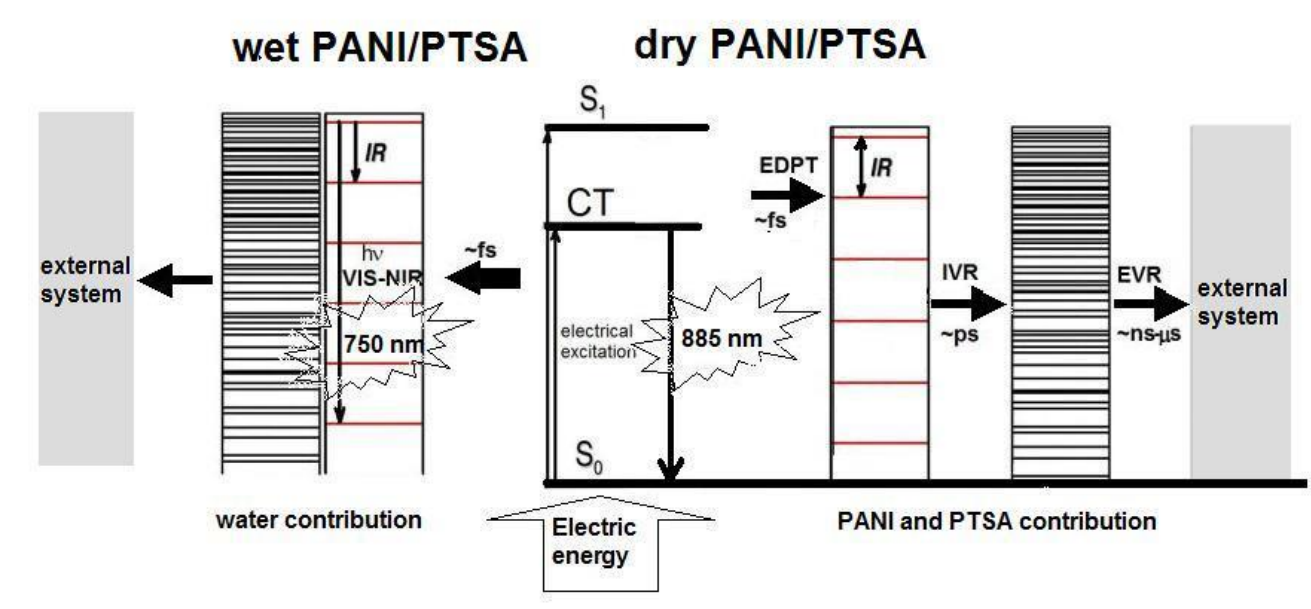

Fig. 1b. Schematic representation of the energy flow during light emission from wet and dry PANI / PTSA (based on published data $^{10}$ ).

\section{Results and discussion}

This work is devoted to unique properties of the system (polyaniline doped with p-toluenesulfonic acid, PANI/PTSA), where electrons and protons are excited when electrically powered, emitting the light in different range. The curiosity is, that the protonic system starts to be active (effective in emission) in the presence of water and it is competitive despite emitting the light of higher photon energy (blue shifted).

The diode formed with dry polyaniline doped with $\mathrm{p}$ toluenesulfonic acid, emits mostly in near infrared (NIR) with maximum at $840-885 \mathrm{~nm}$ (Fig. 1a, 2a). This corresponds to the excitations of $\pi$-electrons and emission due to the charge-transfer (CT) processes in organic materials ${ }^{7-9}$. Here, $\mathrm{CT}$ between PTSA and polyaniline, but also between quinoid and aromatic moieties in polyaniline chain are to be considered.

PTSA can interact with PANI in two ways:

due to Coulomb forces of negative and positive charges of anionic $-\mathrm{SO}_{3}{ }^{-}$and cationic $-\mathrm{N}-\mathrm{H}^{+}$groups, respectively (Fig. 3a, TPSA2 and TPSA3);

- due to non-polar forces, originating from interactions of ?]-electron aromatic rings (Fig. 3a, TPSA1), which are particularly adequate for charge-transfer process; in addition these are responsible for lowering the energy of electron excited state and the energy of photons generated, leading to emission in NIR region with maximum at $885 \mathrm{~nm}$ (electro luminescence of PANI/ $\mathrm{HCl}$, polyaniline doped with $\mathrm{HCl}$ - with no ?-electron interactions, is observed as broad bands at $460 \mathrm{~nm}, 575$ $\mathrm{nm}$ and $657 \mathrm{~nm}$, with maximum at $575 \mathrm{~nm}^{1}$ ).
With increasing the water content (humidity of the active material polyaniline doped with $\mathrm{p}$-toluenesulfonic acid), one can observe a shift in the maximum emission towards the blue and an increase in the light intensity in this part of the spectrum (about $750 \mathrm{~nm}$ ), while the intensity of the emission at $845-885 \mathrm{~nm}$ clearly decreases (Fig. 1a, 2a-c).

This indicates a fast and effective transfer of the energy (provided by the electric current) from the electron system of polyaniline into protonic one (Fig. 1b). Such processes, the ultra fast $(\sim \mathrm{fs})$ electron-to-proton system energy transfer and fast ( ps) flow of energy in the $\mathrm{H}$-bonding network of water, and in consequence the relaxation of electron excitations through coupled hydrogen bonding ( $\sim \mathrm{ns} / \mathrm{ms})$, are well known $^{11,12}$ and studied in detail ${ }^{13,14}$. The energy transfer to the protonic system and accumulation (dissipation in coupled hydrogen bonding system ${ }^{14-16}$ ) is fast and effective enough, that finally, the loss of energy is lower than in the case of the excited polyaniline electron states (Fig. 1b), and the light emission from an excited protonic system ${ }^{4}$ is more efficient. This leads to higher intensity of the light emission with maximum at $750 \mathrm{~nm}$, assigned to protonic system, and lowering the emission at $\sim 850 \mathrm{~nm}$ observed for dry material, when mainly the electrons are excited.

The provided electrical energy excites the hydrogen bonding system in water up to the energy levels of combination vibrations and overtones ${ }^{4}$. The presence of water is crucial. The emission from excited protonic system (water doped with PTSA) clearly dominates in experiments performed with wet PANI/PTSA (Fig. 2 a-c).

The emission spectrum consists of two components: the contribution of excited electron system of dry PANI/PTSA and the excited protonic system (in wet PANI/PTSA), which are additive on a competitive way (Fig. 1a, Fig. 2 a-c). In both cases, despite the emission of light, the dissipation of the excitation energy also takes place in non-radiative processes. This is particularly effective when the electron and proton systems are involved at a comparable level in consuming the 
supplied energy, i.e. for the amplitude ratio of $A_{750 \mathrm{~nm}} / A_{850 \mathrm{~nm}}$ (or $A_{850 \mathrm{~nm}} / A_{750 \mathrm{~nm}}$ ) equal to 1 (Fig. $2 \mathrm{c}$ ). The proton system is not yet ready to compete, and both channels dissipate energy, leading to comparable, lowest light emission efficiency.
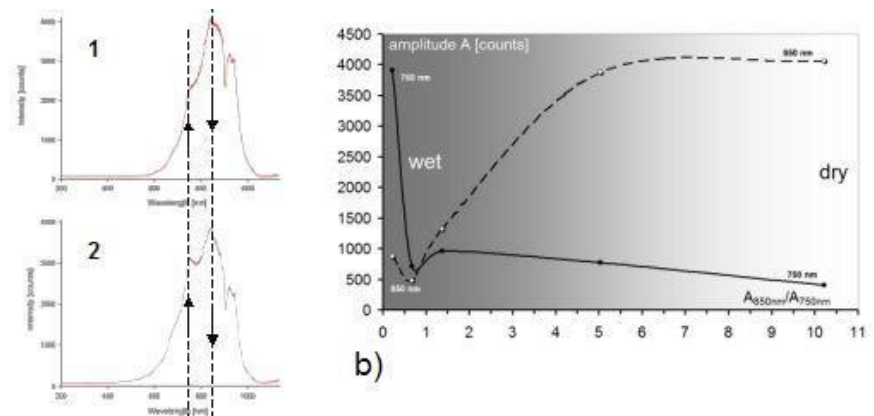

b)
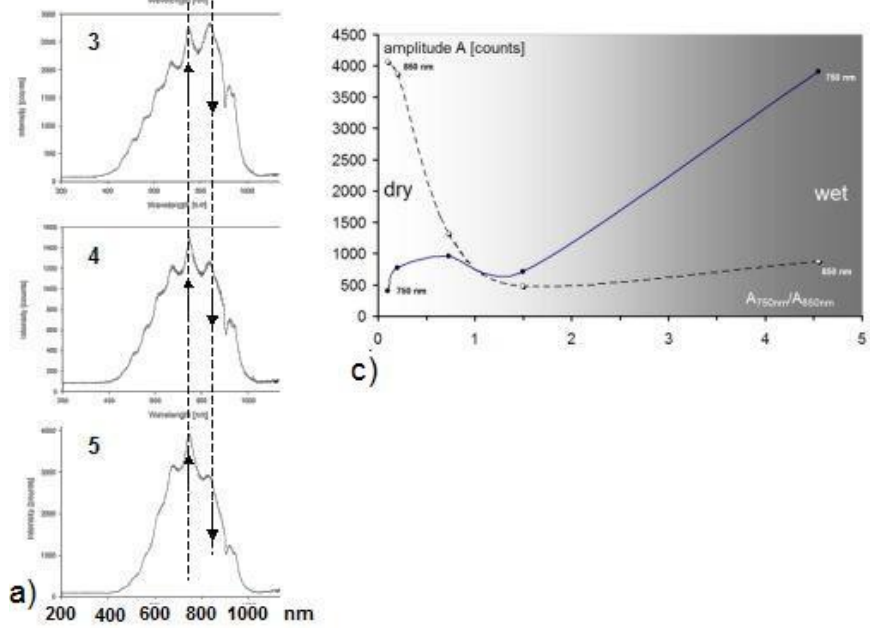

c)

Fig. 2. Evolution of PANI / PTSA electroluminescence spectra with increasing water content (humidity): sample 1 is the driest, sample 5 - the wettest (a). Changes in the spectral amplitude of emission at $750 \mathrm{~nm}$ and $850 \mathrm{~nm}$ as a function of the amplitude ratio of $A_{850 \mathrm{~nm}}$ and $A_{750 \mathrm{~nm}}$ (treated as a measure of the water content in the samples: the emission at $750 \mathrm{~nm}$ is typical for wet PANI/PTSA, and at $850 \mathrm{~nm}$ corresponds to dry PANI/PTSA). Graph in a function of $A_{850 \mathrm{~nm}} / A_{750 \mathrm{~nm}}$ for a better presentation of dry samples (b). Graph in a function of $A_{750 \mathrm{~nm}} / A_{850 \mathrm{~nm}}$ for wetter samples (c).

There is a strong coupling between electrons and protons in polyaniline (including water absorbed ${ }^{11}$ ) - protonation essentially influences its electron system and the electrical conductivity (e.g. emeraldine salt, Fig. 3b). This enables easy energy transfer and excitation the protonic system, when polyaniline is excited owing to the electric current flow.

The quantum energy of photons with the wavelength of 885 $\mathrm{nm}$, of $1.401 \mathrm{eV}$, well corresponds to the polaron band, but also to the energy gap in pernigraniline $(1.2 \mathrm{eV})$, which is smaller by vibrational energy of the para-disubstituted aromatic rings in polyaniline chain $\left(1621 \mathrm{~cm}^{-1}, 0.201 \mathrm{eV}\right)-$ the photon energy of $1.401 \mathrm{eV}$ includes this vibration energy.

Despite the fact that the polyaniline used is a highly conductive material in the form of emeraldine salt with the energy gap of 0.2-0.5 eV and the electrical conductivity of 0.8-3.8 S/cm (what is confirmed directly and adequate to FTIR absorption, Fig. 4), pernigraniline fraction (Fig. $3 \mathrm{c}$ ) can exist inside as domains, which play the role of emission centres. A mixed complex domain structure of polyaniline was discussed previously ${ }^{17}$. High electrical conductivity of polyaniline as a bulk is necessary to reach the threshold current for emission, which is of 7.7A at $3.04 \mathrm{~V}$, and then the working current, which is of $18 \mathrm{~A}$ minimum, at the voltage of $3.84 \mathrm{~V}$ (Fig. 5). Switching ON the emission is sharp and reversible with the voltage change within $0.8 \mathrm{~V}$. The process is reproducible. The electrical conductivity of PANI/PTSA changes from $0.7-1.0 \mathrm{~S} / \mathrm{cm}$ to $3.7-$ $3.8 \mathrm{~S} / \mathrm{cm}$ (during light emission).

Despite sudden changes in the current (and the electrical conductivity, e.g. $0.76 \mathrm{~S} / \mathrm{cm}, 3.8 \mathrm{~S} / \mathrm{cm}$, and $1.05 \mathrm{~S} / \mathrm{cm}$ - before, during and after emission, respectively), the material stay relatively stable in respect to its electrical properties after emission (Fig. 5), including the characteristic parameters measured using the Electron Paramagnetic Resonance (EPR). The EPR signals are very strong for all samples examined (Fig. 6), which indicate on high concentration of polarons (dominating charge carriers in polyaniline). Each of the spectra consists of single narrow Lorentzian line, with asymmetric factor between 1.01 (for crushed dry PANI/PTSA pellets) and 1.16 (for crushed wet PANI/PTSA pellets). The values of gfactor lie in a narrow range from 2.00291 (crushed wet pellets PANI/PTSA) to 2.00299 (crushed dry pellets PANI/PTSA) and correspond to free electrons. The EPR line width after emission is almost the same for dry and wet crushed pellets $(\Delta \mathrm{H}[\mathrm{mT}]=$ 0.236 and 0.228 , respectively) and a bit lower than for starting material $(\Delta \mathrm{H}[\mathrm{mT}]=0.285)$. Thus, the electron interactions did not change essentially.

Interestingly, fast energy transfer from electron to water proton system in wet PANI/PTSA (fs time scale, Fig. 1b), protects against non-radiative energy dissipation, because polarons and bipolarons, generated in polyaniline electron system, are non light-emitting quasi-particles and the energy can be dissipated through the external vibrations (ns- $\mu$ s time scale). In contrast, the excitation of protons is efficient in light emission, as previously observed ${ }^{4}$. There is a surprising but convincing similarity between the electro luminescent spectrums of water doped with sulfonated polystyrene ${ }^{4}$ and the contribution of protonic system near $750 \mathrm{~nm}$ in present experiments with wet PANI/PTSA (Fig. 6). Thus, this process is fast enough ( $p$ s or less time scale) to be competitive effectively with non-radiative dissipation of energy (ns-ms time scale), Fig. $1 b$.

Due to effective electrons-to-protons energy transfer and excitation the protonic system, lower dissipation of the energy provided leads to emission the photons of higher energy with higher efficiently than the excited polyaniline electron system can do it, when electrically powered. The difference in photon energy is of $\sim 0.2 \mathrm{eV}$, what corresponds to excitation the 
molecular vibrations in polyaniline, including aromatic rings (near $1600 \mathrm{~cm}^{-1}$ ) and quinoid grouping $\left(1516 \mathrm{~cm}^{-1}\right)^{18,19}$. These are strongly influencing the electron energy, but are not involved in the excitation of the proton system, so the energy is not absorbed and dissipated this way, leading finally to a blue shift of the emission spectrum in the presence of water (wet PANI/PTSA).

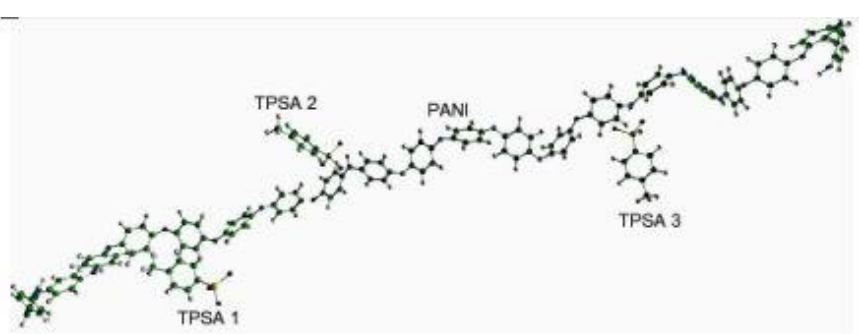

a)

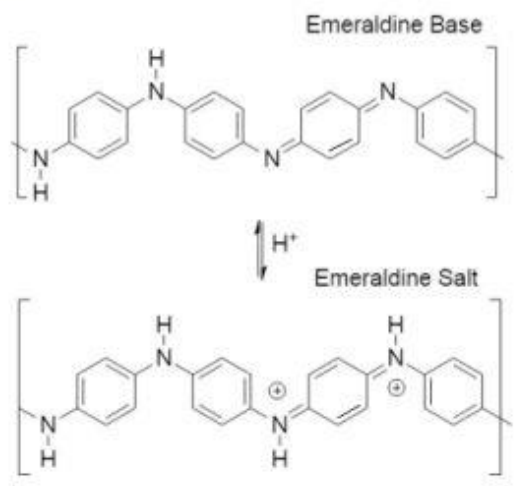

b)

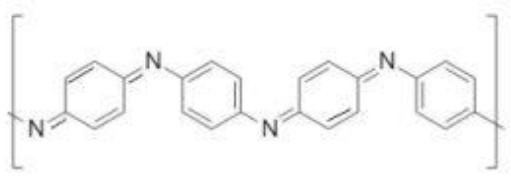

c)

Fig. 3. A model of PANI / PTSA complex (a); emeraldine base and emeraldine salt (b); pernigraniline (c).

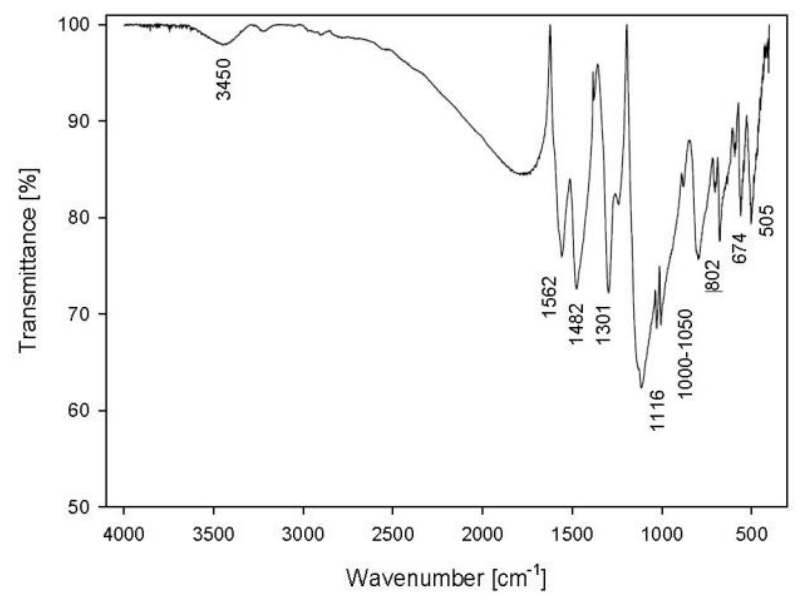

Fig. 4. FTIR spectrum of PANI / PTSA.
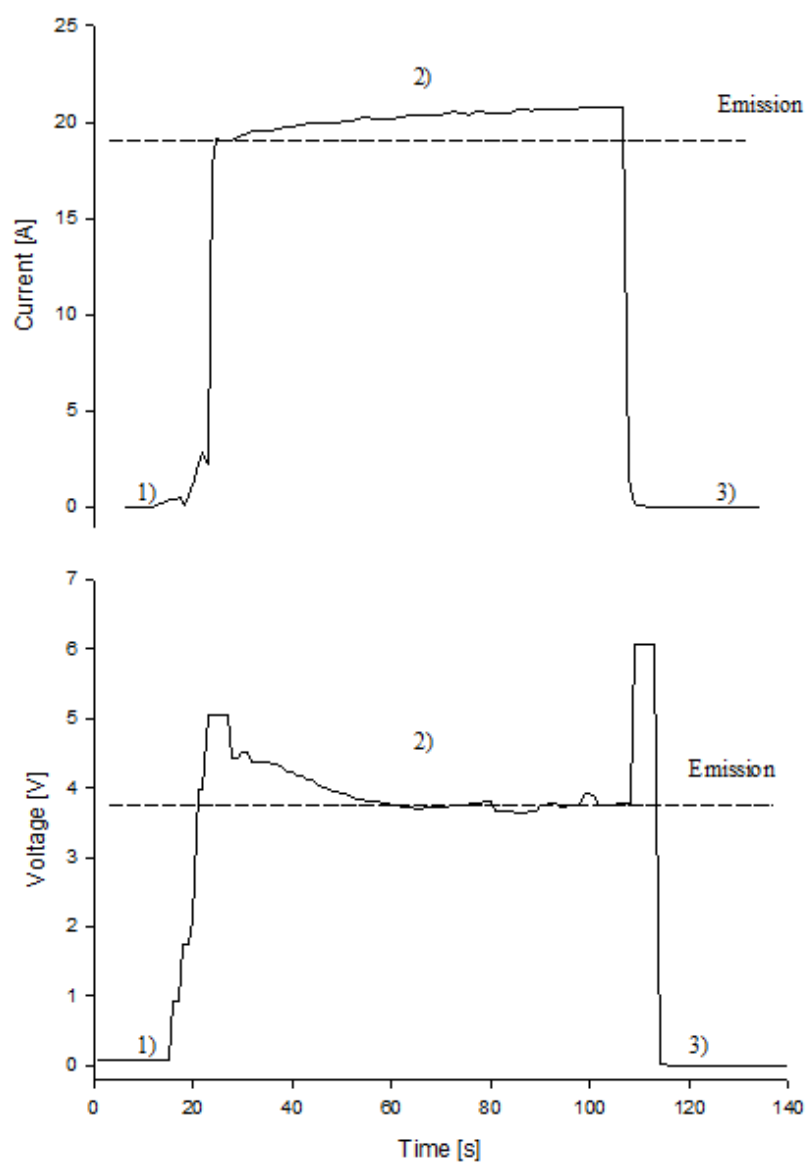

Fig. 5. Typical current and voltage changes as a function of time, recorded before (1), during (2) and after the emission (3) for PANI/PTSA; the dashed line shows the minimum operating voltage of $3.74 \mathrm{~V}$ and the current of $18 \mathrm{~A}$. 
a)
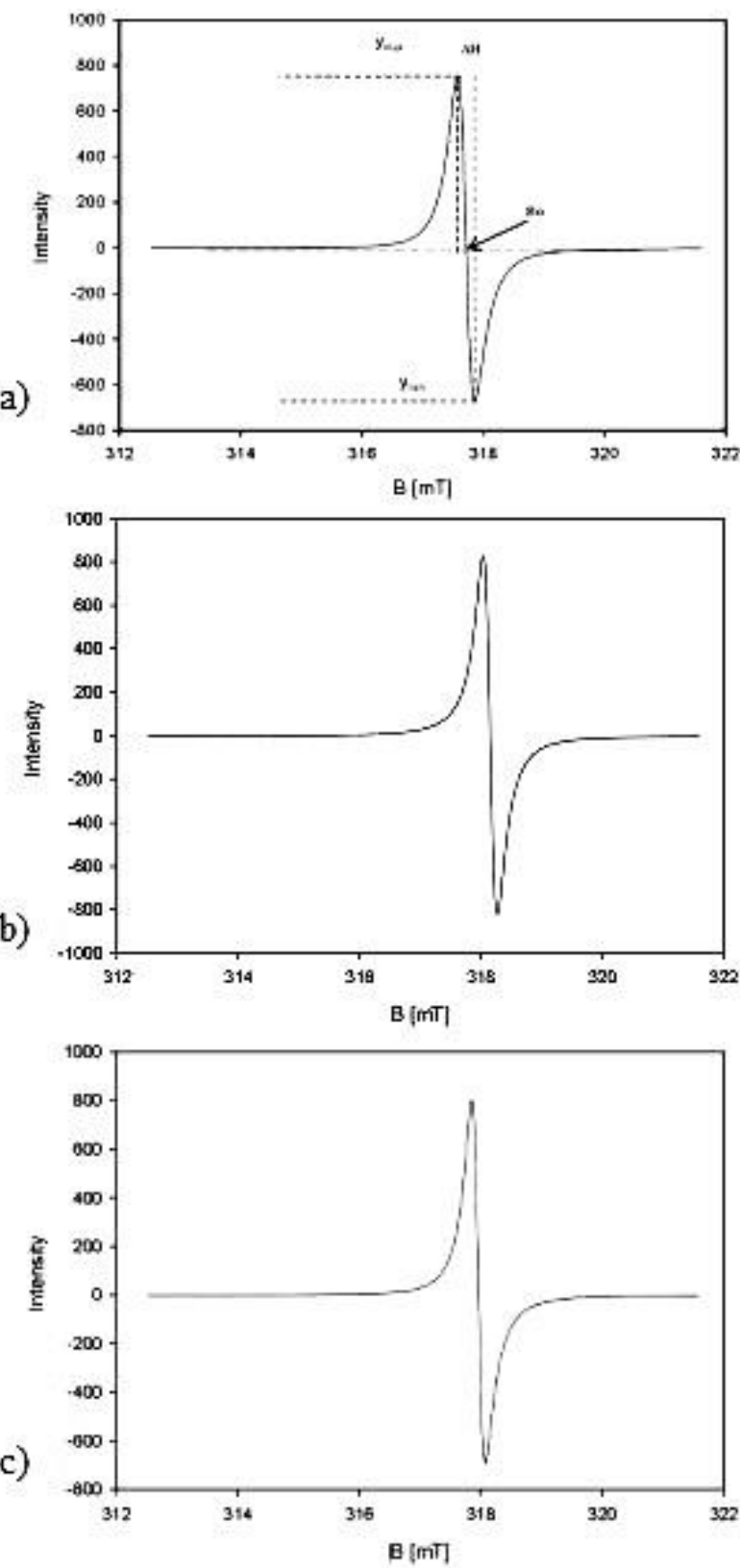

Fig. 6. The EPR spectra of PANI/PTSA:

a) PANI/PTSA before experiments (powder), g-factor $=2.00292$, linewidth $\Delta \mathrm{H}[\mathrm{mT}]=0.285$, asymmetry $A=1.13$;

b) crushed dry pellets of PANI/PTSA after light emission, g-factor $=2.00299$, linewidth $\Delta H[\mathrm{mT}]=$ 0.236 , asymmetry $A=1.01$

c) crushed wet pellets of PANI/PTSA after light emission, g-factor $=2.00291$, linewidth $\Delta \mathrm{H}[\mathrm{mT}]=$ 0.228 , asymmetry $A=1.16$

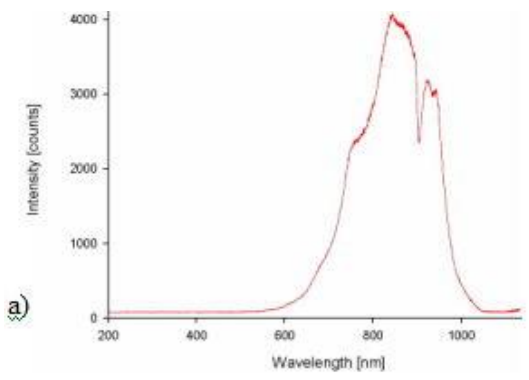

b)

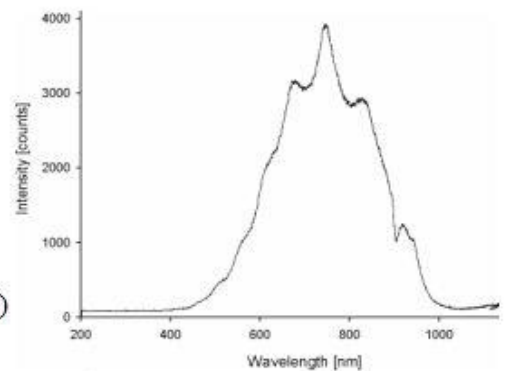

c)

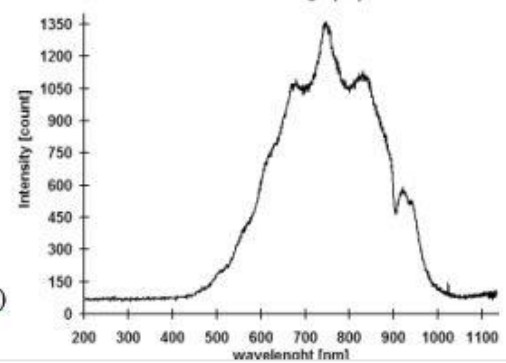

Fig.7. PANI /PTSA electroluminescence spectra in the dry state (a) and in the presence of water (b), and emission from water doped with sulfonated polystyrene ${ }^{4}$ (c).

\section{Experimental}

\section{Materials \& Methods}

Polyaniline was prepared by oxidation of aniline hydrochloride ( $10 \%$ in water at $\mathrm{pH}$ about 1 ) with chemical method described elsewhere, modified in our laboratory.

The polymeric material (polyaniline) was characterized with physical and chemical methods (FTIR, EPR, elemental analysis, electrical conductivity measurements) giving results similar to the values measured for materials previously prepared in our laboratory ${ }^{1,2,20}$. 
Chemicals and solvents:

Hydrochloric acid $\mathrm{HCl}$ (Stanlab, pure p.a.) - $600 \mathrm{ml} 1 \mathrm{M}$ solution prepared from concentrated hydrochloric acid (36\%) - $50 \mathrm{ml}$ $36 \% \mathrm{HCl}$ dissolved in $550 \mathrm{ml} \mathrm{H} 2 \mathrm{O}$.

Aniline hydrochloride $\mathrm{C}_{6} \mathrm{H}_{8} \mathrm{NCl}$, (Fisher Scientific, pure p.a.) $11.4 \mathrm{~g}$ aniline hydrochloride dissolved in $300 \mathrm{ml}$ of $1 \mathrm{M}$ hydrochloric acid.

Ammonium persulfate $\left(\mathrm{NH}_{4}\right)_{2} \mathrm{~S}_{2} \mathrm{O}_{8}$ (Chempur, pure p.a.) $-6.9 \mathrm{~g}$ of ammonium persulfate was dissolved in $200 \mathrm{ml}$ of $1 \mathrm{M}$ hydrochloric acid.

Ammonium hydroxide $\mathrm{NH}_{4} \mathrm{OH}$ solution $25 \%$ (POCh, pure p.a.) $500 \mathrm{ml} 1 \mathrm{M}$ solution prepared from concentrated ammonium hydroxide (25\%) - $37 \mathrm{ml} 25 \%$ ammonium hydroxide dissolved in $463 \mathrm{ml} \mathrm{H} \mathrm{H}_{2} \mathrm{O}$.

Chloroform $\mathrm{CHCl}_{3}$ (Chempur, pure p.a.) $-75 \mathrm{ml}$.

p-Toluenesulfonic acid $\mathrm{CH}_{3} \mathrm{C}_{6} \mathrm{H}_{4} \mathrm{SO}_{3} \mathrm{H}$ (Aldrich, p.a.) - $1.4 \mathrm{~g}$ of $\mathrm{p}$ toluenesulfonic acid was dissolved in $75 \mathrm{ml}$ of chloroform.

\section{Polyaniline emeraldine base, PANIEB}

Aniline hydrochloride $(6.9 \mathrm{~g})$ was dissolved in $300 \mathrm{ml}$ of $1 \mathrm{M}$ hydrochloric acid. At the same time, $11.4 \mathrm{~g}$ of ammonium persulfate was dissolved in $200 \mathrm{ml}$ of $1 \mathrm{M}$ hydrochloric acid (separately).

The ammonium persulfate solution was slowly added to a solution of the aniline hydrochloride. The resulting dark green solution was stirred with a magnetic stirrer at room temperature for 24 hours.

The precipitate was filtered under reduced pressure and washed several times with distilled water, until the filtrate was nearly colourless and neutral. The solid product was treated with $500 \mathrm{ml}$ of $1 \mathrm{M}$ ammonium hydroxide and stirred on a magnetic stirrer at room temperatures for 20 hours.

The precipitate was isolated by filtration under reduced pressure and washed several times with distilled water until $\mathrm{pH}^{\sim 7}$. The dark blue precipitate of emeraldine base (PANIEB) was dried at ambient conditions (yield $1.8 \mathrm{~g}$ ).

Elemental analysis:

Found [\%] C 74.05, H 5.01, N 13.61, O 7.33

Calculated [\%] C 72.69, H 5.09, N 14.13, 08.07

$\left[\mathrm{C}_{\mathbf{1 2}} \mathrm{H}_{\mathbf{8}} \mathrm{N}_{\mathbf{2}} \cdot \mathrm{H}_{\mathbf{2}} \mathbf{O}\right]_{\mathrm{n}}$, where one molecule of $\mathrm{H}_{2} \mathrm{O}$ is associated with two monomeric units of: $\mathrm{C}_{6} \mathrm{H}_{4} \mathrm{~N}$.

Polyaniline protonated with p-toluenesulfonic acid, PANI/PTSA $1.4 \mathrm{~g}$ of $\mathrm{p}$-toluenesulfonic acid was dissolved in $75 \mathrm{ml}$ of chloroform. Then, $0.9 \mathrm{~g}$ of PANIEB was added to this solution in small portions. The mixture was stirred with a magnetic stirrer for two hours. The dispersion was dripped into a beaker containing $200 \mathrm{ml}$ of distilled water while stirring all the time. The precipitate was filtered under reduced pressure and washed several times with distilled water until $\mathrm{pH} \sim 4,5$ has been obtained. To remove most water from the product was washed several times with methanol. The obtained polyaniline protonated with p-toluenesulfonic acid (PANI/PTSA) was dried for 24 hours at room temperature.
Elemental analysis:

Found [\%] C 61.605, H 5.145, N 8.47, O 18.528, S 6.252

Calculated [\%] C 60.450, H 5.280, N 8.46, O 19.330, S 6.460

[3 $\left.\left[\mathrm{C}_{6} \mathrm{H}_{4} \mathbf{N} \cdot \mathrm{H}_{2} \mathrm{O}\right] \cdot \mathrm{C}_{7} \mathrm{H}_{8} \mathrm{SO}_{3}\right]_{n}$ ), where three molecules of $\mathrm{H}_{2} \mathrm{O}$ are associated with three monomeric units of the acid: $\mathrm{CH}_{3} \mathrm{C}_{6} \mathrm{H}_{4} \mathrm{SO}_{3} \mathrm{H}$.

FTIR spectra (Fig. 5; B- benzenoid rings, Q- quinoid rings):

$3450 \mathrm{~cm}^{-1} \mathrm{~N}-\mathrm{H}$ stretching mode,

1562 and $1482 \mathrm{~cm}^{-1} \mathrm{C}=\mathrm{C}$ stretching of quinoid and benzenoid rings,

$1301 \mathrm{~cm}^{-1} \mathrm{C}-\mathrm{N}$ stretching of secondary aromatic amine,

$1116 \mathrm{~cm}^{-1} \mathrm{C}-\mathrm{H}$ in-plane bending vibration (mode of $\mathrm{N}=\mathrm{Q}=\mathrm{N}$, $\mathrm{Q}=\mathrm{N}+\mathrm{H}-\mathrm{B}$, and $\mathrm{B}-\mathrm{N}+\mathrm{H}-\mathrm{B})$,

$802 \mathrm{~cm}^{-1}$ out-of-plane deformation of $\mathrm{C}-\mathrm{H}$ in 1,4 -substituted benzene ring,

$505 \mathrm{~cm}^{-1} \mathrm{C}-\mathrm{H}$ deformation of an aromatic ring.

$1000-1050 \mathrm{~cm}^{-1}$ and at $674 \mathrm{~cm}^{-1},-\mathrm{SO}_{3}^{-}$vibrations.

Elemental analyses were performed using a model Vario EL III elemental analyser (Elementar Analysensysteme $\mathrm{GmbH}$, Germany).

FTIR spectra were recorded using the spectrometer model IFS 66/s (Bruker, USA).

The Electron Paramagnetic Resonance (EPR) spectra were recorded using an EPR spectrometer model SE/X 2547 (RADIOPAN, Poland).

The emission UV-VIS NIR spectra were registered on-line with Spectrometer model 2000 Ocean Optics PC2000 at a resolution of $0.5 \mathrm{~nm}$, at the same time, when the current and the voltage were measured.

The beam profile was pictured directly with digital camera: Pentax Kr 12.4 MP + 18-55 mm lens.

The measurements were performed at ambient conditions and in a dark room.

The samples, formed at a pressure up to $6000 \mathrm{kG} / \mathrm{cm}^{2}$ as pellets of thickness $0.4-0.5 \pm 0.01 \mathrm{~mm}$ and diameter $3 \mathrm{~mm}$, were placed in a measuring holder, inside in a glass tube of a wall thickness of 1-2 $\mathrm{mm}$, between two solid copper electrodes of a diameter of $4.5 \mathrm{~mm}$ (or one of $4.5 \mathrm{~mm}$ and the second of $3 \mathrm{~mm}$ ) and a length of $25 \mathrm{~mm} .{ }^{1,2,4}$

The voltage and the current were measured with an accuracy at least of $0.1 \%$, using Brymen digital multimeter, model BM859s (computer controlled) and Metrahit Energy Multimeter (computer controlled) with precision standard resistor $0,001 \Omega$ for current measurement, respectively. Stabilized power supplies applied: INCO Z-3020 - DC voltage source, adjustable between 0.1-30 V under load current 0-20 A and INCO Z-5001 - DC voltage source, adjustable between 0.1$500 \mathrm{~V}$ under load current 0-1 A.

To register simultaneously, the light beam and the emission spectrum, the optical fibre of the spectrometer was mounted on the same side as the photo camera, in most cases parallel to the optical axis of the camera (another configuration was also used). The distance between the sample and the aperture of the optical fibre was $1-3 \mathrm{~cm}$, and the camera was located at a distance of $15-20 \mathrm{~cm} .{ }^{4}$ 


\section{Conclusions}

The effective electrons-to-protons energy transfer and excitation the protonic system (resulting in lower dissipation the energy provided), lead to emission the photons of higher energy and with higher efficiently than the excited polyaniline electron system can do it (for wet PANI/PTSA), when electrically powered. The water proton system effectively competes with the electron system in the wet PANI/PTSA, capturing the supplied electrical energy, which results in intense light emission with a blue shift.

\section{Author Contributions}

$K R$ and EF are responsible for most of the experiments, data collection and partial interpretation.

SG recorded EPR spectra.

$\mathrm{JJL}$ is the author of the general concept, responsible for evaluating and interpreting the experimental results and writing the manuscript.

\section{Conflicts of interest}

There are no conflicts to declare.

\section{Acknowledgements}

We wish to thank the Centre for Advanced Technology AMU in Poznań for hiring laboratory space.

\section{Notes and references}

1 J. J. Langer, B. Miładowski, S. Golczak, K. Langer, P. Stefaniak, A. Adamczak, M. Andrzejewska, L. Sójka and M. Kalisz, J. Mater. Chem., 2010, 20, 3859.

2 M. Kalisz, S. Golczak, E. Frąckowiak, K. Langert and J. J. Langer, J. Mater. Chem. C, 2016, 4, 6634.

3 J. Siedlecka, J. J. Langer, UV-VIS-NIR emission of polypyrrolegraphite diod electrically powered. Prequel Symposium on Advanced Technologies and Materials, preATAM2020, 13 15.10. 2020, PORT, Wrocław, Poland.

4 J.J. Langer, E. Frąckowiak, S. Golczak, J. Mater. Chem. C, 2020, 8, 943.

5 J.J. Langer, Applied Physics A, 1984, 34, 195.

6 J.J. Langer, Applied Physics A, 1985, 38, 59.

7 D. C. A. Valente, M. T. do Casal, M. Barbatti, T. A. Niehaus, A. J. A. Aquino, H. Lischka, and Th. M. Cardozo, J. Chem. Phys., 2021, 154, 044306.

8 I. Bargigia, L. R. Savagian, A. M. Österholm, J. R. Reynolds and C. Silva, J. Am. Chem. Soc. 2021, 143, 1, 294.
9 A. Shahalizad, A. Malinge, L. Hu, G. Laflamme, L. Haeberlé, D. M. Myers, J. Mao, W. G. Skene, S. Kéna-Cohen, Adv. Funct. Mater. 2021, 31, 2007119.

10 A.L. Sobolewski, W. Domcke, Phys. Chem. Chem. Phys. 2010, 12, 4897.

11 A.L. Sobolewski, W. Domcke, ChemPhysChem, 2006, 7, 561.

12 S. Perun, A.L. Sobolewski, W. Domcke, J. Phys. Chem. A, 2006, 110, 9031.

13 K. L. Corp, E. J. Rabe, X. Huang, J. Ehrmaier, M. E. Kaiser,A. L. Sobolewski, W. Domcke, and C. W. Schlenker, J. Phys. Chem. C, 2020, 124, 9151.

14 A.L. Sobolewski, W. Domcke, J. Phys. Chem. A 2007, 111, 11725.

15 H. Elgabarty, T. Kampfrat, D. J. Bonthuis, V. Balos, N. K. Kaliannan, Ph. Loche, R. R. Netz, M. Wolf, T. D. Kühne, M. Sajadi, Sci. Adv. 2020; 6 : eaay7074.

16 J. Zhou, S. Lin, H. Zeng, J. Liu, B. Li, Y. Xu, X. Zhao and G. Chen, Mater. Horiz., 2020, 7, 2936.

17 J.J. Langer, Synthetic Metals, 1990, 36, 35.

18 M. Arora and S.K. Gupta, CPl 075, Perspectives in Vibrational Spectroscopy: ICOPVS 2008; edited by V. K. Vaidyan and V. S. Jayakumar, American Institute of Physics; AIP Conference Proceedings, 2008, 118, 1075.

19 M.-I. Boyer, S. Quillard, E. Rebourt, G. Louarn, J. P. Buisson, A. Monkman, and S. Lefrant, J. Phys. Chem. B 1998, 102, 7382.

20 J. J. Langer, Solid State Commun., 1978, 26, 839. 